\title{
VACUUM SYSTEM FOR THE ACCELERATING STRUCTURE OF THE IFUSP MICROTRON
}

\author{
J. Takahashi, M. N. Martins* , L. Portante, R. da R. Lima, Laboratório do Acelerador Linear \\ Instituto de Física da Universidade de São Paulo, CP 66318, 05315-970 São Paulo, SP, Brazil \\ F. T. Degasperi, Faculdade de Tecnologia de São Paulo, FATEC-SP
}

\section{Abstract}

This paper describes the vacuum system adopted for the accelerating structures of the IFUSP Microtron accelerator. The structure uses the Los Alamos sidecoupled cavities and presents a vacuum port at each coupling cavity. We describe the vacuum system, the advantages of the adopted configuration, some construction details and calculations on the expected performance.

\section{INTRODUCTION}

The Laboratório do Acelerador Linear do Instituto de Física da Universidade de São Paulo (LAL-IFUSP) finished the construction of a $\beta=1$ continuous wave accelerating structure [1] for the IFUSP microtron [2], which presented excellent properties [3]. The structure is a 1.04-m long standing wave, side coupled (SCS) design, with 17 accelerating cavities and 16 coupling cavities.

One of the main advantages of the side-coupled accelerating structure, as compared to other structure designs, is the possibility of pumping each of the coupling cavities. This is an important feature, for it allows a quick pumping of the degassing produced by RF heating or sparking in the structure. Our structure presents 16 coupling cavities, each of them connected by a tube with a conductance of $12 \mathrm{l} / \mathrm{s}$. Figure 1 shows a schematic drawing of the structure, with dimensions and details of the cavities.
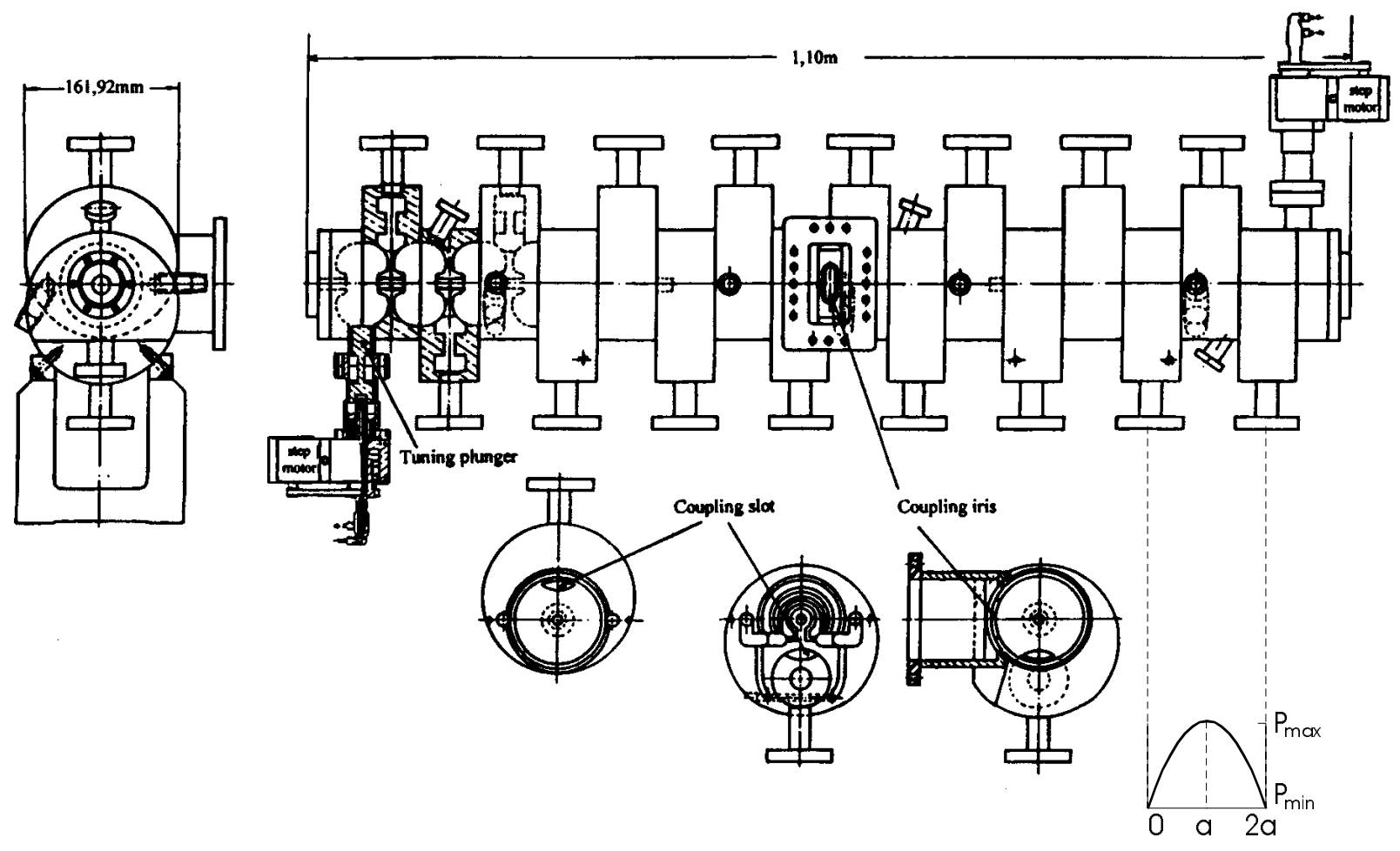

Figure 1: Schematic drawing of the structure, with detail showing the pressure along the length of a "module" (see text).

*E-mail: martins@if.usp.br 
To ease the mechanical design of the vacuum system we decided to use only the ports facing downwards, as shown in Fig. 2, which presents a side view of the structure and the manifold.

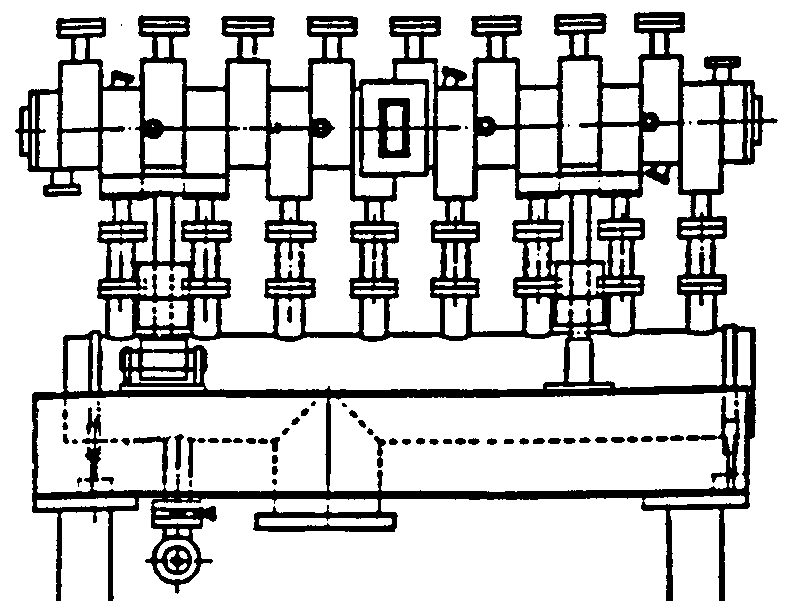

Figure 2: Side view of the structure, showing the coupling with the manifold.

\section{MODELING}

There are two basic options on how to pump the 8 vacuum ports: with 8 vacuum pumps of about $40 \mathrm{l} / \mathrm{s}$ pumping speed, or with a manifold connecting the 8 ports to a single $300 \mathrm{l} / \mathrm{s}$ vacuum pump. Figure 3 shows a schematic drawing of the manifold used.

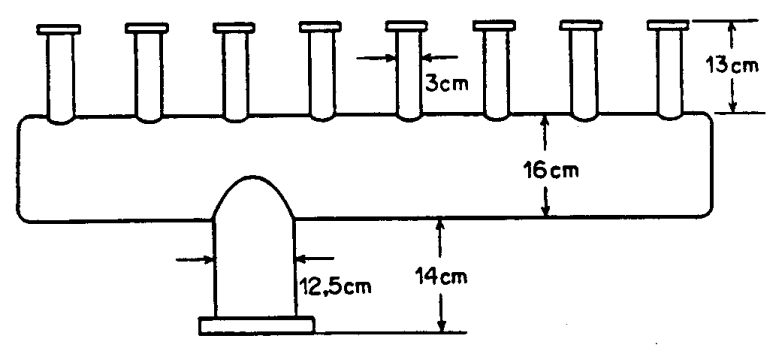

Figure 3: Schematic drawing of the manifold.

A simplified model was used to evaluate the effective pumping speed of the system and also the steady state pressure along the length of the structure. We divided the structure in 8 modules composed of one half of a coupling cavity, two full accelerating cavities, one coupling cavity (facing down) and another one half of a coupling cavity, as depicted in Fig. 1. We then proceeded to evaluate the conductance and the degassing of the main parts of this module. Beginning with the conductance, to pump the gas in the furthermost volume, the coupling cavity facing up, it has to go through the accelerating cavity, down to the coupling cavity facing down, to the exit tube, to the connecting tube of the manifold, the manifold itself and finally to the pump. In the evaluation of the pressure profile along the structure, we need the effective pumping speed at the accelerating cavity, because that is where the electric field is most intense.

The effective pumping speed can then be written as

$\frac{1}{S_{\text {eff }}}=\frac{1}{C_{T}}+\frac{1}{S_{p}}$

where $S_{\text {eff }}$ is the effective pumping speed at the accelerating cavity, $S_{p}$ is the pumping speed of the vacuum pump and $C_{T}$ is the total conductance of the system. $C_{T}$ can be calculated by

$$
\frac{1}{C_{T}}=\frac{1}{C_{W}}+\frac{1}{C_{C C}}+\frac{1}{C_{E T}}+\frac{1}{C_{C T}}+\frac{1}{C_{M}}+\frac{1}{C_{P T}}
$$

where $C_{W}$ is the conductance of the window between the coupling and accelerating cavities (approximated by an ellipse shape), $C_{C C}$ the conductance of the coupling cavity, $C_{E T}$ the conductance of the exit tube, $C_{C T}$ the conductance of the connecting tube (between the exit tube and the manifold), $C_{M}$ the conductance of the manifold, and $C_{P T}$ the conductance of the tube that connects the manifold to the vacuum pump. We consider the gas to be $\mathrm{N}_{2}$, at $298 \mathrm{~K}$ and molecular flow. Several simplifications were done, mainly in the geometry of the cavities, which are very complex.

$C_{W}$ was calculated considering the window to be elliptical, with major and minor axes equal to $2.4 \mathrm{~cm}$ and $1.6 \mathrm{~cm}$, respectively. Then $C_{W}=36 \mathrm{l} / \mathrm{s}$. The coupling cavity was approximated by two curved tubes of rectangular cross section, forming a shape close to a toroid, resulting in $C_{C C}=94 \mathrm{l} / \mathrm{s}$. The exit and connecting tubes present the most restricted conductance of the whole system, being narrow (2- and $3-\mathrm{cm}$ in diameter, respectively) and long (5.4- and 20.6-cm long, respectively), resulting in $C_{E T}=12 \mathrm{l} / \mathrm{s}$ and $C_{C T}=11 \mathrm{l} / \mathrm{s}$. The conductance of the manifold will depend on the port which is being pumped. We calculated the two extreme cases $(850$ and $1300 \mathrm{l} / \mathrm{s})$ and used the average, $C_{M}=1075$ $l / \mathrm{s}$. The pumping tube of the manifold presents $C_{P T}=780$ $l / \mathrm{s}$ (which includes the whole distance from the manifold to the ion pump). Then, using Eq. (2), the total conductance of the system is $C_{T}=4.7 \mathrm{l} / \mathrm{s}$. Substituting this value in Eq. (1) and considering $S_{P}=300 \mathrm{l} / \mathrm{s}$, we obtain for the effective pumping speed on each module, at the entrance of the accelerating cavity, $S_{\text {eff }}=4.6 \mathrm{l} / \mathrm{s}$.

If, instead of the manifold, eight $40 \mathrm{l} / \mathrm{s}$ pumps were used, connected at each port by $100-\mathrm{cm}$ long and $7.5-\mathrm{cm}$ in diameter tubes, the effective pumping speed at the accelerating cavity would be $4.9 \mathrm{l} / \mathrm{s}$. The difference is less than $7 \%$. So the effective pumping speed with the manifold and a $300 \mathrm{l} / \mathrm{s}$ pump, is equivalent to eight $40 \mathrm{l} / \mathrm{s}$ pumps, but the manifold solution costs about $30 \%$ of the other. 
To determine the pressure distribution along the structure, we must calculate the degassing of the cavities and the local effective pumping speed. In our simple model, we consider the pumping volume to be that of one accelerating cell plus one half of the volume of the coupling cell and exit tube (closed) facing upwards. This corresponds, in the structure depicted schematically in Fig. 1, to the portion between points $O$ and $a$. The accelerating cavity presents a degassing surface of approximately $190 \mathrm{~cm}^{2}$, while the coupling cell plus exit tube add up to $180 \mathrm{~cm}^{2}$. Since we are considering just one half of this last surface, the total degassing surface will be $280 \mathrm{~cm}^{2}$. Since the window between the coupling and accelerating cavities presents the lowest conductance, to simplify the calculation we will model the system by a cylindrical cavity with internal surface of $280 \mathrm{~cm}^{2}$ over a length $a$, and conductance of $36 \mathrm{l} / \mathrm{s}$, with pumping ports separated by a distance $2 a(a=6$ $\mathrm{cm}$ ). This model presents a quadratic solution that can be written as

$$
p(x)=\frac{-q}{c} x^{2}+\frac{2 q a}{c} x+\frac{q a}{S_{e f f}}, \text { for } 0 \leq x \leq 2 a
$$

Where $p(x)$ is the pressure at the point $x$ along the structure, $q$ is the degassing per unit length, and $c$ is the specific conductance. For our specific conditions, we find $p(0)=3.7 \times 10^{-8}$ Torr and $p(a)=3.9 \times 10^{-8}$ Torr.

\section{CONCLUSIONS}

The adopted configuration worked well. The final pressure at the manifold should be better than $3 \times 10^{-8}$ Torr. The manifold option presents a performance very close to the one using 8 individual pumps, with a cost about $70 \%$ lower. The difference of about $10 \%$ between the maxima and minima of the pressure distribution along the structure is acceptable and supports the decision to close the vacuum ports facing up.

\section{ACKNOWLEDGMENTS}

This work was supported by FAPESP (Fundação de Amparo à Pesquisa do Estado de São Paulo), and CNPq (Conselho Nacional de Desenvolvimento Científico e Tecnológico).

\section{REFERENCES}

[1] L.M. Young and J.M. Potter, "CW side coupled linac for the Los Alamos-NBS racetrack microtron", Los Alamos National Laboratory Report, LA-9324-C, 1982.

[2] J. Takahashi et al., "The IFUSP racetrack microtron", in Proceedings of the $3{ }^{\text {rd }}$ European Particle Accelerator Conference, edited by HHenke and P. J. Genaz, Editions Frontières, France, 1992. Vol. 1, p. 429-431.
[3] J. Takahashi et al., "Plunger frequency control of the side coupled accelerating structure for the IFUSP microtron", in Proceedings of the 1997 Particle Accelerator Conference,

http://www.trimf.ca/pac97/papers/pdf/4p038.pdf. 